\title{
Modal Analysis of a Single-Structure Multiaxis MEMS Gyroscope
}

\author{
Muhammad Ali Shah, ${ }^{1,2}$ Faisal Iqbal, ${ }^{1}$ Ibrar Ali Shah, ${ }^{3}$ and Byeungleul Lee ${ }^{1}$ \\ ${ }^{1}$ School of Mechatronics Engineering, Korea University of Technology and Education (KoreaTech), 1600 Chungjeol-ro, \\ Byeongcheon-myeon, Dongnam-gu, Cheonan-si, Chungcheongnam-do 330-708, Republic of Korea \\ ${ }^{2}$ Shinsung Advanced Institute (Shinsung CઐT), Gasan 2-ro, Geumcheon-gu, Seoul, Republic of Korea \\ ${ }^{3}$ Department of Computer Software Engineering, University of Engineering and Technology Peshawar, \\ Mardan Campus, Khyber Pakhtunkhwa, Pakistan \\ Correspondence should be addressed to Byeungleul Lee; bllee@koreatech.ac.kr
}

Received 30 July 2016; Revised 11 October 2016; Accepted 16 November 2016

Academic Editor: Oleg Lupan

Copyright (C) 2016 Muhammad Ali Shah et al. This is an open access article distributed under the Creative Commons Attribution License, which permits unrestricted use, distribution, and reproduction in any medium, provided the original work is properly cited.

\begin{abstract}
This paper reports on designing a single-structure triaxes MEMS capacitive gyroscope which is capable of measuring the three angular velocities on a single drive. A Z-shaped beam for the support of folded coupling spring has been applied to suppress the unwanted mode and decrease the stress effect at the spring ends. The unique coupling spring has changed the driving motion, due to which slide film damping in the driving mode has been reduced. This reduction can lead to higher performance of the sensor with less requirements on vacuum level which decreases the cost of fabrication. Simulation analysis has been performed in COMSOL Multiphysics and Matlab Simulink to finalize the design for fabrication. After finite element analysis, the driving, $x$-sensing, $z$-sensing, and $y$-sensing are, respectively, found to be $13.30 \mathrm{KHz}, 13.40 \mathrm{KHz}, 13.47 \mathrm{KHz}$, and $13.51 \mathrm{KHz}$.
\end{abstract}

\section{Introduction}

Most of today's inertial sensors are based on microelectromechanical systems (MEMS). Due to the use of MEMS technology, the contemporary sensors are of light weight in design, low cost, and compact size. With MEMS, sensors have the added advantage of achieving high performance with low power consumption [1]. MEMS inertial sensors comprise gyroscopes and accelerometers. Accelerometers and gyroscopes play an important role in the MEMS industry among sensors. Gyroscope is used to measure the angular rotations about the three perpendicular axes, that is, $x-, y$-, or $z$-axis, while accelerometer is used to sense the linear acceleration. Gyroscope which is used to measure a single axis rotation is called a single-axis gyroscope while the gyros that are used to sense more than one axis are called multiaxis gyroscopes. Mostly, single-axis gyroscopes are $z$-axis designed with different approaches as identified in the research studies [2-5]. Also, many multiaxis gyroscope designs have been published for sensing two or three axis angular velocities [5-8]. In $[7,8]$, the presented designs need three separate drive voltages as they are based on three drive structures. These designs, however, result in complex circuitry and more power consumption.

Due to small size and high performance, single-structure triaxes gyroscopes are aimed for MEMS sensor industries nowadays. The use of single structure in gyroscopes not only simplifies electronics design but also leads to low power, low cost, and reduced noise in the overall system. These advantages pave ways for wide applications of inertial measurement units (IMUs) in industries, for example, electronic stability control, body motion dynamics, mobile gaming, and image stabilization to name a few.

To win space, most MEMS IMUs designs combine two in-plane accelerometers and gyroscopes ( $x$ - and $y$-axes) with an out-of-plane accelerometer and gyroscope ( $z$-axis), which permits placing all sensors on a single chip [9]. Single drive mass has an advantage to adjust all the gyroscopes $(x-y$-, and $z$-gyros) inside a single structure which has reduced 6 degree-of-freedom (6-DOF) IMU die size and the cost of the MEMS sensing element $[10,11]$.

Motion sensing combo sensors are a hot topic owing to the wide applications of IMUs as well as in terms of market 
potential and competition among the players. Recently, due to the growing trend of 6- and 9-DOF devices applications, the field leaders (e.g., STMicroelectronics, Bosch, Invensense, $\mathrm{AKM}$, and mCube to name a few) have devised innovative technical solutions, to have the right patents $[12,13]$ to protect their inventions.

For improving the IMUs performance and size reduction, a number of different designs have been published targeting single-structure triaxes MEMS gyroscopes [13-16]. The study of [13] suggests an integrated single drive three-axis MEMS gyroscope with improved driving structure where the improved driving movements have been achieved by using folded coupling springs. Coupling drive masses ensure their synchronous movement with a given phase due to elastic coupling elements. For coupling of drive masses and enabling their synchronous movement with each other, the authors in [15] suggested U-shaped synchronizing springs near the center of the device. The study of [16] has proposed a vibratory ring spring in their presented design which makes all sensing schemes necessary for 3-axis angular rate. Another single-structure triaxis gyro design has been patented in [17]; however, the proposed gyro design has used the anchor in the coupling spring system which can have anchor losses during the drive-mode operation. Furthermore, the use of these anchors also increases the complexity and fabrication steps.

The conflict between STMicroelectronics and Invensense has also opened new ways for the researchers in terms of single-structure triaxes gyroscopes. For example, a singlestructure 3-axis gyroscope has been analyzed to reduce the drive-force coupling by using secondary auxiliary mass [18]. In [19], authors have proposed a single-drive mass gyroscope with a high resonant frequency.

In MEMS motion sensors design, elastic elements play very significant role. This is due to the fact that the designed spring beams, being able to deliver the desired transduction, can transfer vibration and bring improvement in the impact to the system. Since, the gyroscope is affected by the external forces and acceleration, therefore, it becomes pivotal to provide the elastic coefficient of the vibration system along with alleviation of the external impact by the spring beams. For this purpose, a number of studies have been done on the elastic elements [20, 21]. In [21], the authors have analyzed the performance of commonly used springs in MEMS sensor technology.

Folded springs have been used in many published MEMS inertial sensors designs due to the low cross-axis errors [22] and perfect motions of the moving parts of those sensors. Also, in single-structure MEMS gyroscope designs, the folded or U-shaped spring is used mostly for the coupling of the drive masses. Joining the individual drive masses into single one requires coupling spring. Almost, all the previous suggested designs propose the usage of same typical coupling spring. For example, the designs proposed in $[15,16]$ suggest the use of U-shaped coupling springs. However, U-shaped and folded coupling springs result in higher stress fracture as compared to the new unique coupling spring suggested in this paper, which is attached at two points with the driving mass. Due to single point of attachment of U-shaped and folded coupling springs to the driving mass, they receive large stress effect at the end points (Figure 3(b)) which can lead to the spring breakage during the operation.

In this paper, a single-structure triaxes MEMS gyroscope has been proposed with a unique coupling spring. The Zshaped beam has been introduced for the support of the folded spring and to suppress the unwanted mode. The coupling spring is composed of folded spring supported by a Zshaped beam. The work presented in this letter is an extension of our previous study [23] in which the design has been thoroughly analyzed and results have been presented after extensive simulation of the design in COMSOL Multiphysics and Matlab Simulink.

\section{Structural Analysis of the Proposed Design}

The proposed design is a single-structure triaxial MEMS capacitive gyroscope with the dimension of $1428 \mu \mathrm{m} \times 1428 \mu \mathrm{m}$. The device is anchored at five positions. The gyroscope comprises four driving masses coupled by the four coupling spring as shown in Figure 1. The proposed design is capable of measuring pitch, roll, and yaw angular velocities where electrode plates can be placed on the substrate beneath the $x$ - and $y$-sensing proof masses. With the applied roll angular velocity, $\Omega x$, to the MEMS gyroscope, electrodes beneath the $y$-sensing proof masses are used to sense the applied roll angular velocity, $\Omega x$. Similarly, the pitch angular velocity, $\Omega y$, is sensed by utilizing the electrodes beneath the $x$-sensing proof masses. Moreover, for sensing yaw angular velocity, each of the $Z$-sense masses includes $Z$-sense movable and fixed electrodes. To find the yaw angular velocity, $\Omega z$, the changes in capacitance between fixed and movable electrodes are measured. When a yaw angular velocity, $\Omega z$, is applied to the MEMS gyroscope, the two $Z$-sensing masses move in $y$-direction opposite to each other. In this way, a change in capacitance occurs between fixed and movable electrodes. Figure 1 shows the proposed gyroscope, containing the fixed parts and moving mass. A further simplified model of the proposed gyroscope is represented in Figure 2. In Figure 2, $K_{c}$ represents the coupling spring, which is used to couple the driving masses. The simulation results of the resonant frequencies from COMSOL are shown in Figure 3.

2.1. Coupling Spring. The spring beams, which deliver the desired transduction, play an important role in MEMS gyroscope design. The presented gyroscope has more elastic elements which have been properly distributed in the design to make all the motions useful. Almost the same folded coupling spring has been used in the prior published gyroscope designs. The proposed design contains a unique coupling spring which comprises a Z-shaped beam and folded spring.

A Z-shaped beam is introduced for the support of the folded spring. The coupling spring has the following effects:

(i) It reduces stress on the spring ends as the spring is attached at two points with the driving mass.

(ii) It has changed the driving mass motion which has a reduced slide film damping effect in the outside static and moving driving electrodes. 


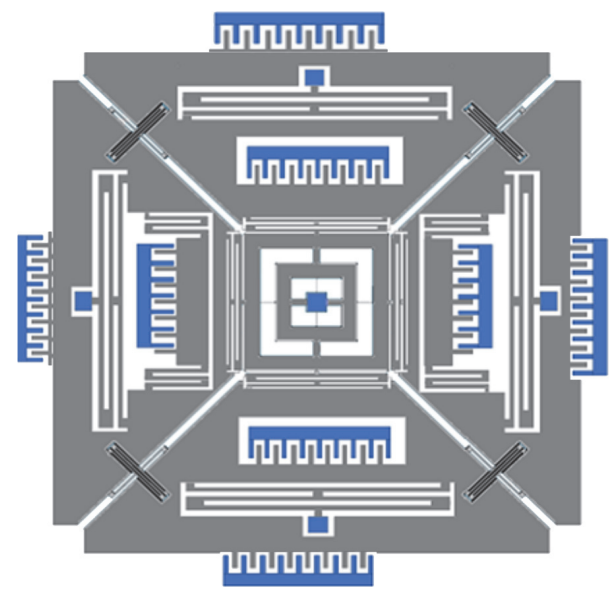

Moving part

Fixed part

FIGURE 1: Representation of a 2D diagram of the proposed gyroscope.

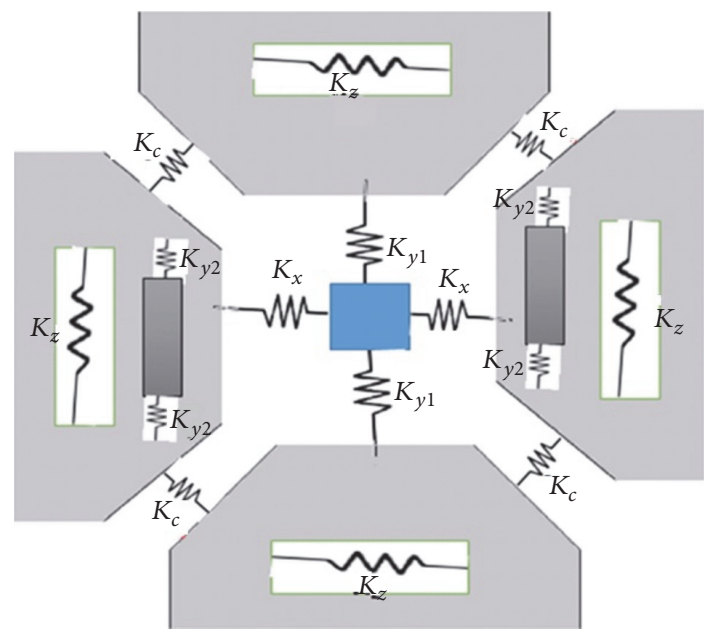

FIgURE 2: Simplified model of the proposed gyroscope design.

(iii) Due to the use of the Z-shaped beam with the folded spring, the unwanted mode has been suppressed.

2.2. Effect of Z-Shaped Beam. The effect of coupling spring in the design has been analyzed. The design is simulated with and without Z-shaped beam. The differences between the final required mode and the unwanted mode (next to the final required mode) were compared in both spring cases. The design with Z-shaped beam in the coupling spring $(S 1)$ was found to have large frequency difference $(\Delta f)$ between the final required and unwanted frequency modes as compared to the design without the Z-shaped beam in the coupling spring (S2). The frequency difference between the required and unwanted modes is, respectively, $2072 \mathrm{~Hz}$ and $825 \mathrm{~Hz}$ of the designs with and without Z-shaped beams. On the basis of this analysis, the design with the Z-shaped beam for the support of the folded spring is better as compared to the design without the Z-shaped beam, as it suppressed the unwanted mode shown in the design without the Zshaped beam. Moreover, the coupling spring with Z-shaped beam has a less stress effect at the spring ends attached to the drive mass as compared to the design without the $\mathrm{Z}$ shaped beam in the coupling spring as shown in Figure 4. The reason behind the less stress effect is that the $\mathrm{Z}$-shaped beam coupling spring is attached at a two points to the drive mass. The two designs with different coupling springs and the resonant frequencies are shown in Figure 5 (design with Z-shaped beam in coupling spring) and Figure 6 (design without Z-shaped beam in coupling spring). Figure 7 shows the graph of frequency difference between two modes of the design with two different coupling springs $S 1$ and $S 2$.

\section{Slide Film Damping Estimation in Driving Mode}

Generally, damping due to the surrounding air can be ignored for a conventional mechanical machine because the energy dissipated by air damping is much smaller than the energy dissipated by other mechanisms. However, air damping can affect micromechanical devices; therefore, the energy consumed by air damping must be minimized to maximize motion of the mechanical part with a supply of limited energy.

In micromachined vibratory systems, such as gyroscopes, two damping effects can be found generally, that is, slide and squeeze film damping. Squeeze film damping is mostly due to the out-of-plane motion of the gyroscope while; on the other side, the slide film damping is due to the lateral movement of the gyroscope. The fluid flow is governed by the NavierStokes equation. The equations of damping, $c$, calculated by [24] from the Navier-Stokes equation are given below:

$$
\begin{aligned}
c & =\frac{\mu A}{h}, \\
c_{\mathrm{combs}} & =\frac{2 n A_{\mathrm{combs}} \mu}{g_{c}+2 \lambda} .
\end{aligned}
$$

Equation (1) represents the damping coefficients for both fluid velocities $u$ and $v$ for no-slip boundary conditions while (2) is for the slip-boundary conditions. In (1), $n$ is the total number of combs, $A_{\text {combs }}=t \times L_{c}$ is overlapping area of each pair of combs, $g_{c}$ is the gap between the two combs, and $\lambda$ is the mean free path of the molecules of fluid.

In the proposed design, the driving motions of the two pairs of drive masses are in opposition to each other; that is, when one pair moves towards center of the gyroscope then the other pair moves away from the center and vice versa. Due to this drive mass movement, the overlapping area of the electrodes attached to one pair decreases while the other pair increases. This effect results in overall decrease of slide film damping [17] as compared to the prior published referred designs. Figure 8 shows the proposed gyroscope design driving masses moving configuration with thickness, $t$, and the overlap length of the combs, $L_{c}$. 


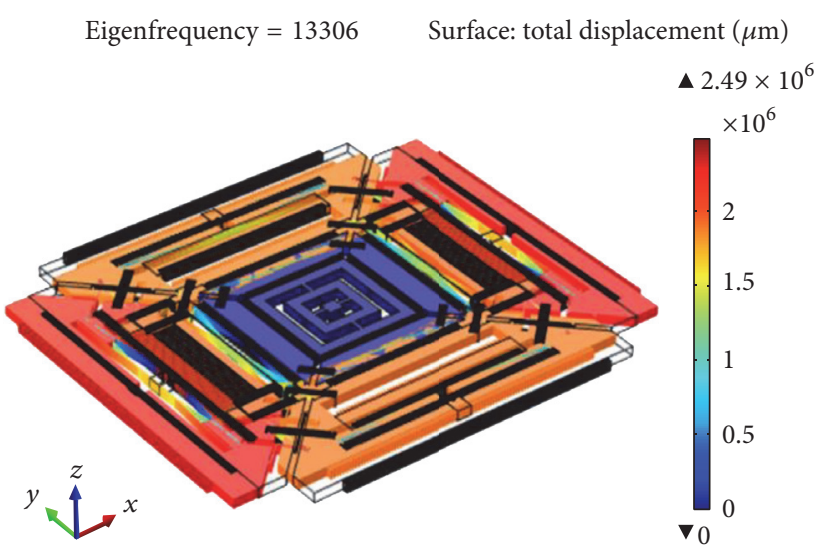

(a)

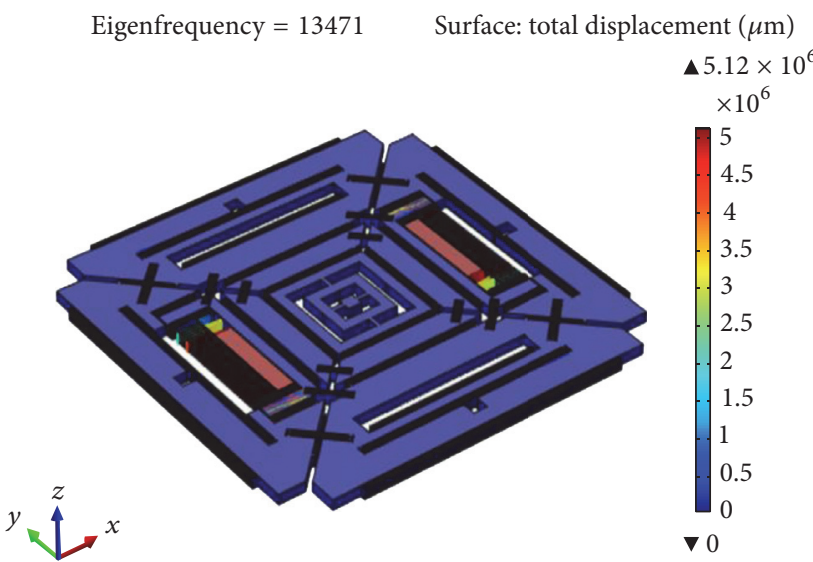

(c)

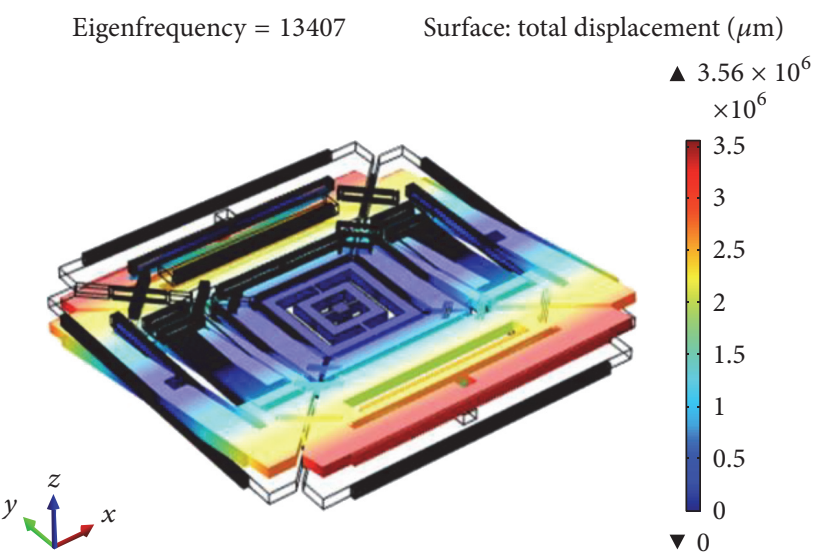

(b)

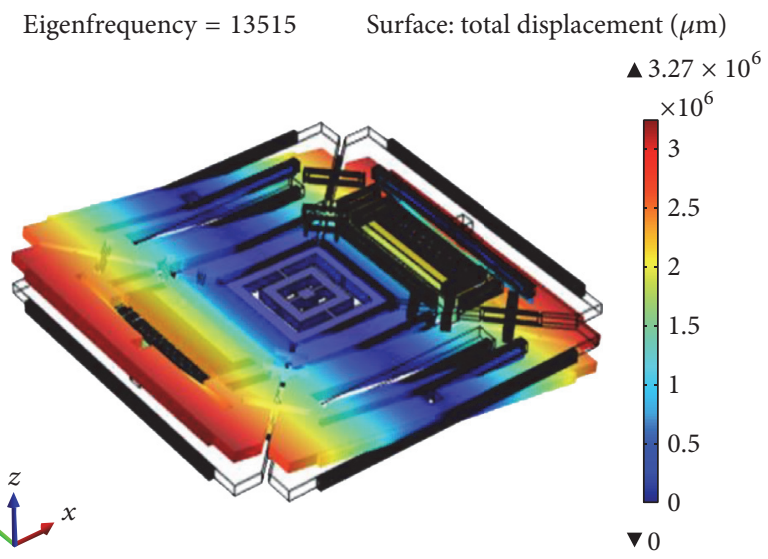

(d)

FIGURE 3: Required resonant four modes of the proposed design. (a) Driving mode, (b) $X$-sensing mode, (c) $Z$-sensing mode, and (d) $Y$ sensing mode.

TABLE 1: Electrical parameters of the proposed design.

\begin{tabular}{lcc}
\hline Types & Notation & Capacitance \\
\hline Driving & $C_{\mathrm{DRV}}$ & $758 \mathrm{fF}$ \\
Drive sense & $C_{\mathrm{DRS}}$ & $503 \mathrm{fF}$ \\
Coriolis sense $X$-gyro & $C_{\mathrm{CSX}}$ & $794 \mathrm{fF}$ \\
Coriolis sense $Y$-gyro & $C_{\mathrm{CSY}}$ & $794 \mathrm{fF}$ \\
Coriolis sense $Z$-gyro & $C_{\mathrm{CSZ}}$ & $521 \mathrm{fF}$ \\
\hline
\end{tabular}

\section{Matlab Simulations}

Simulations of the drive and sense-mode operations of the proposed design have been performed in Matlab Simulink. Due to simple implementation and zero steady state error, automatic gain control (AGC) with PI is commonly used for the self-oscillation of gyroscope [25]. A self-oscillating closed loop, utilizing PI controller, was simulated using Matlab Simulink. The electrical and mechanical parameters used during the simulation are shown in Tables 1, 2, and 3. The block diagram self-oscillating control loop is shown in Figure 9.
The closed loop transfer function of the self-oscillation loop is given by

$$
H(s)=\frac{\left(\omega_{0} / 2 k\right) K_{\mathrm{tot}} H_{\mathrm{cntrl}}(s) V_{\mathrm{AC}}}{S+\omega_{0} / 2 Q+\left(\omega_{0} / 2 k\right) K_{\mathrm{tot}} H_{\mathrm{cntrl}}(s) V_{\mathrm{AC}}} .
$$

Figure 10 shows the drive sense voltage of a single drive three axis gyroscope. The settling time was $0.7 \mathrm{sec}$ at optimized values. The settling time can be further reduced by increasing the gain, which will result in high overshoot.

An open loop sense detection scheme was also simulated where the quadrature error was neglected for simplification and the individual charge amplifiers were used for the detection. Since the potential application for the proposed gyroscope is consumer electronics, angular rate of $5 \mathrm{dps}$ at different amplitude was applied. As the input rate is amplitude modulated signal at the resonance frequency, a coherent demodulator was used to detect the corresponding input rate as shown in Figure 11. 
Eigenfrequency $=12235$ Surface: von Mises stress $\left(\mathrm{N} / \mathrm{m}^{2}\right)$

$\Delta 1.05 \times 10^{14}$

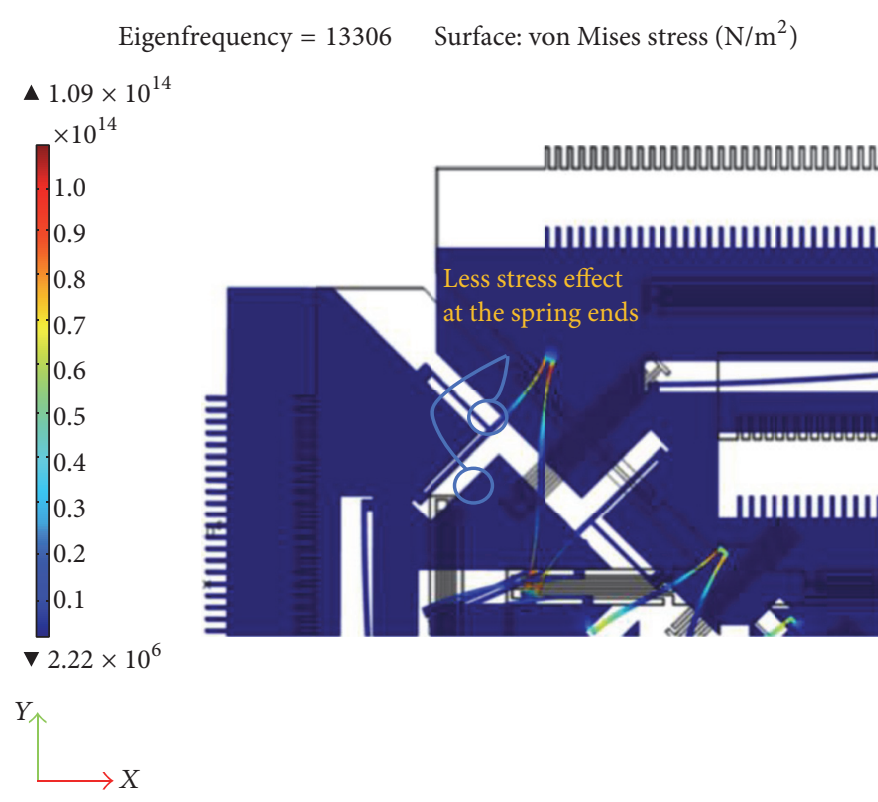

(a)
FIGURE 4: Stress effects at the spring ends of coupling springs. (a) Design with Z-shaped spring in the coupling spring. The stress effect is reduced at the spring ends attached to the driving mass. (b) Design without the Z-shaped beam in the coupling spring. A large stress effect occurs when von Mises stress has been applied during the drive mode.

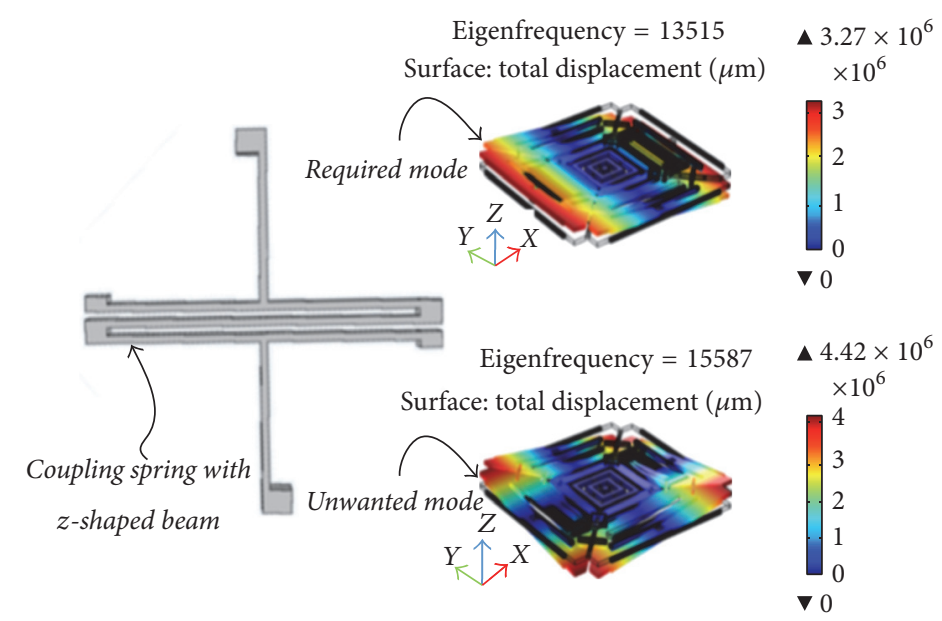

FIGURE 5: Design with Z-shaped beam in the coupling spring (S1). Final required mode frequency is $13515 \mathrm{~Hz}$ while the unwanted mode (next to the final required mode) frequency is $15587 \mathrm{~Hz}$, which has a difference of $2072 \mathrm{~Hz}$.

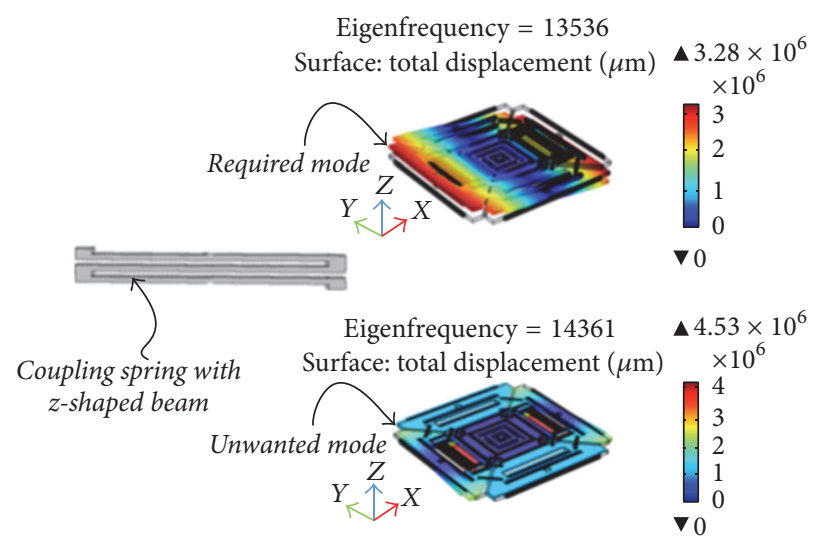

FIGURE 6: Design without Z-shaped beam in coupling spring (S2). Final required mode frequency is $13536 \mathrm{~Hz}$ while the unwanted mode (next to the final required mode) frequency is $14361 \mathrm{~Hz}$, which has a difference of $825 \mathrm{~Hz}$. 


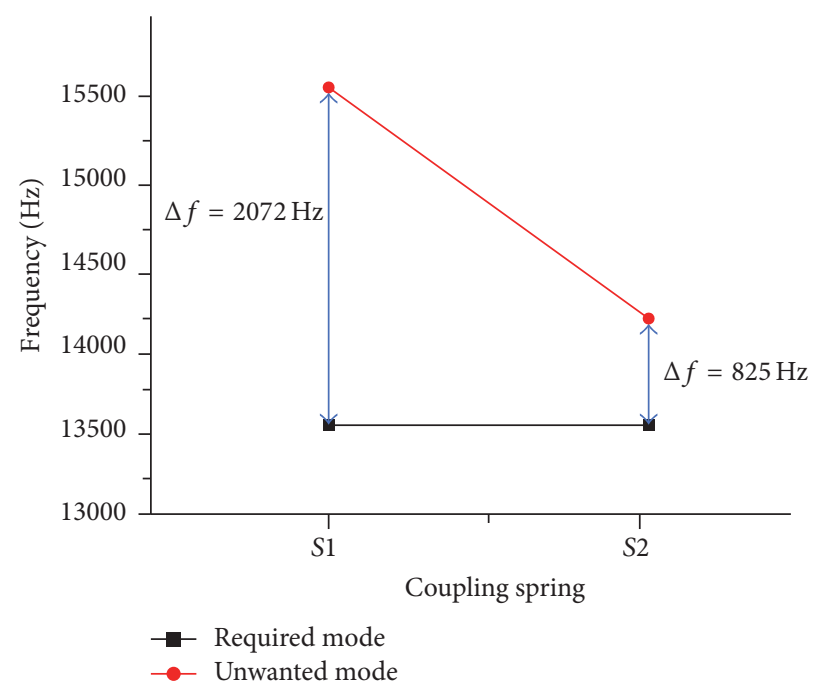

FIGURE 7: This figure shows the two designs with two mentioned coupling springs. $S 1$ shows the design with coupling spring that contains $\mathrm{Z}$-shaped beam while $S 2$ shows the design without the Z-shaped beam in the coupling spring. The frequency difference between the required mode and the unwanted mode in case of $S 1$ is $2072 \mathrm{~Hz}$ which is higher than the frequency difference of $S 2$.

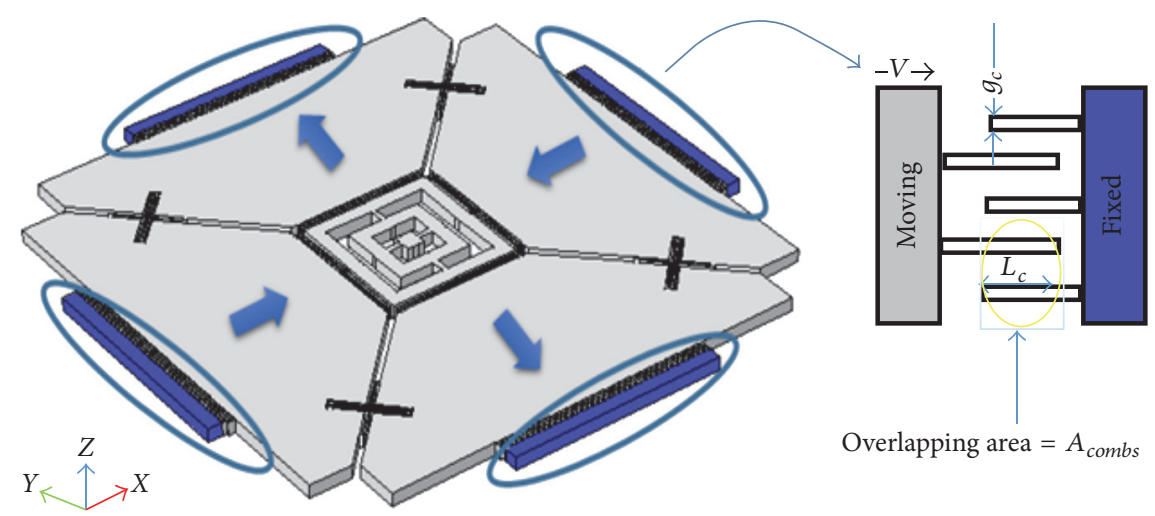

FIGURE 8: Driving masses motion configuration of the proposed design. When two masses move outside, then the other two masses move to the center of the device.

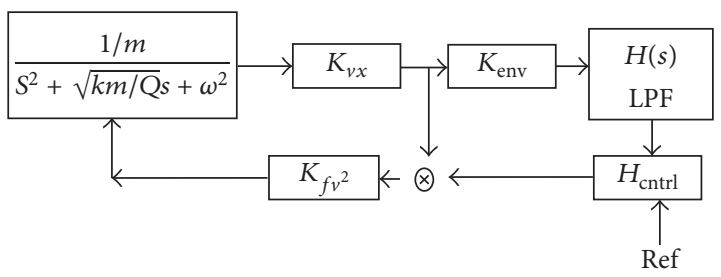

FIGURE 9: Block diagram of self-oscillating control loop.

\section{Conclusion and Future Work}

In this work, our focus was on the small-footprint and bigdata requirement, which has been achieved by designing and simulating a single-structure triaxes gyroscope. The dimension of the device is $1428 \mu \mathrm{m} \times 1428 \mu \mathrm{m}$ which is capable of measuring three mutual perpendicular axes. Due to the use of the unique coupling spring, the driving motions of the two pairs of drive masses are in opposition to each other; that is, when one pair moves towards center of the gyroscope, then

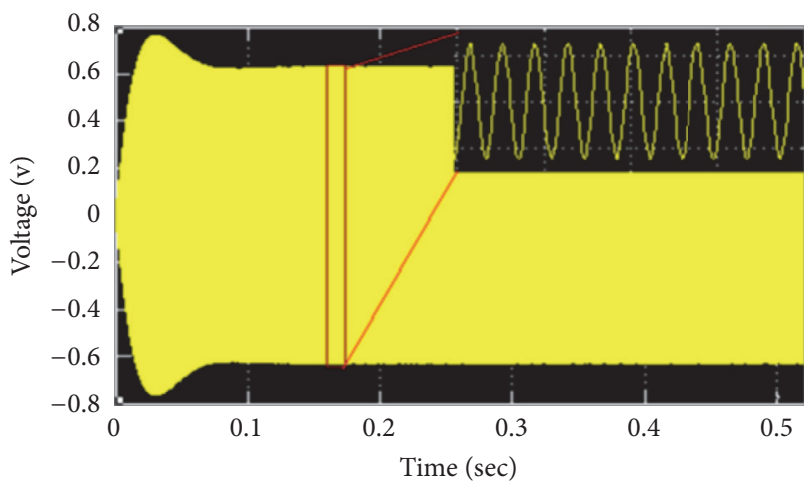

Figure 10: Drive sense voltage.

the other pair moves away from the center and vice versa. Due to this drive mass movements, the overlapping area of the electrodes attached to one pair decreases while the other pair increases resulting in an overall decrease in slide film 


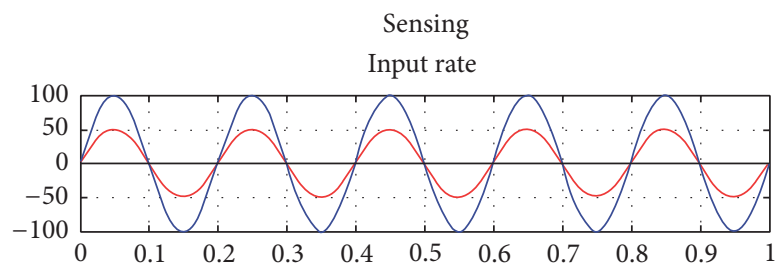

(a)

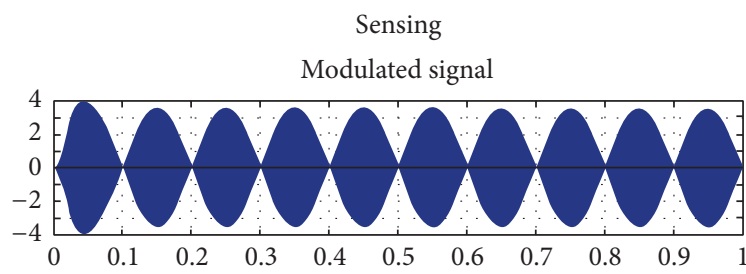

(b)

Sensing

Output rate

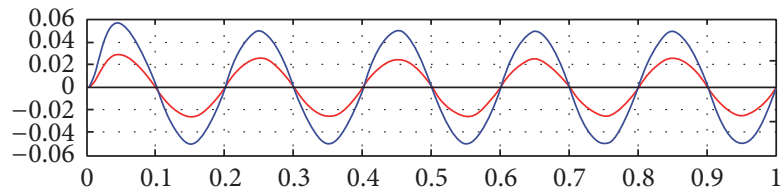

(c)

Figure 11: Individual rate detection. (a) Input rate. (b) Modulated Signal. (c) Output rate.

TABLE 2: Mechanical driving parameters of the proposed design.

\begin{tabular}{lcccc}
\hline & & \multicolumn{2}{c}{ Driving parameters } & \\
Frequency $\left(f_{d}\right)$ & Stiffness $\left(K_{d}\right)$ & Mass & Drive quality factor & Coupling spring \\
Lidth
\end{tabular}

TABLE 3: Mechanical sensing parameters of the proposed design.

\begin{tabular}{|c|c|c|c|c|c|}
\hline \multicolumn{6}{|c|}{ Sensing parameters } \\
\hline \multicolumn{6}{|c|}{ Sense quality factor $=2500$} \\
\hline \multicolumn{6}{|c|}{$X$-gyro } \\
\hline \multirow{2}{*}{ Frequency $\left(f_{x}\right)$} & \multirow{2}{*}{$\begin{array}{l}\text { Change in frequency } \\
\qquad\left(f_{x}-f_{d}\right)\end{array}$} & \multirow{2}{*}{ Stiffness $\left(K_{x}\right)$} & \multirow{2}{*}{ Moment of inertia $\left(I_{x}\right)$} & \multicolumn{2}{|c|}{$X / Y$-gyro sensing spring } \\
\hline & & & & Length & Width \\
\hline $13407 \mathrm{~Hz}$ & $101 \mathrm{~Hz}$ & $91.8 \times 10^{-6} \mathrm{Nm}$ & $12.95 \times 10^{-15} \mathrm{kgm}^{2}$ & $776 \mu \mathrm{m}$ & $3.5 \mu \mathrm{m}$ \\
\hline \multicolumn{6}{|c|}{$Y$-gyro } \\
\hline \multirow{2}{*}{ Frequency $\left(f_{y}\right)$} & \multirow{2}{*}{$\begin{array}{l}\text { Change in frequency } \\
\qquad\left(f_{y}-f_{d}\right)\end{array}$} & \multirow{2}{*}{ Stiffness $\left(K_{y}\right)$} & \multirow{2}{*}{ Moment of inertia $\left(I_{y}\right)$} & \multicolumn{2}{|c|}{$X / Y$-gyro sensing spring } \\
\hline & & & & Length & Width \\
\hline $13515 \mathrm{~Hz}$ & $209 \mathrm{~Hz}$ & $93.9 \times 10^{-6} \mathrm{Nm}$ & $12.95 \times 10^{-15} \mathrm{kgm}^{2}$ & $776 \mu \mathrm{m}$ & $3.5 \mu \mathrm{m}$ \\
\hline \multicolumn{6}{|c|}{ Z-gyro } \\
\hline \multirow{2}{*}{ Frequency $\left(f_{z}\right)$} & \multirow{2}{*}{$\begin{array}{l}\text { Change in frequency } \\
\qquad\left(f_{z}-f_{d}\right)\end{array}$} & \multirow{2}{*}{ Stiffness $\left(K_{z}\right)$} & \multirow{2}{*}{ Mass } & \multicolumn{2}{|c|}{$Z$-gyro sensing spring } \\
\hline & & & & Length & Width \\
\hline $13515 \mathrm{~Hz}$ & $209 \mathrm{~Hz}$ & $43.8 \mathrm{~N} / \mathrm{m}$ & $6.13 \times 10^{-9} \mathrm{~kg}$ & $174 \mu \mathrm{m}$ & $3 \mu \mathrm{m}$ \\
\hline
\end{tabular}

damping. This reduction can lead to the high performance of the sensor with less requirement on vacuum level which decreases the cost of fabrication of MEMS gyroscope. The proposed design is simulated in COMSOL Multiphysics and Matlab Simulink. A Z-shaped beam has been introduced in the coupling spring for the support of the folded spring. The driving, $x$-sensing, $z$-sensing, and $y$-sensing are, respectively, found to be $13.30 \mathrm{KHz}, 13.40 \mathrm{KHz}, 13.47 \mathrm{KHz}$, and $13.51 \mathrm{KHz}$.

The device is under fabrication process. After fabrication, the testing will be performed for measuring the real quality factor, scale factor, bias stability, resolution, and bandwidth in our future study. Furthermore, instead of using individual charge amp, we are working to develop schemes for using a single charge amplifier in the sense circuit.

\section{Competing Interests}

The authors declare that they have no competing interests.

\section{Acknowledgments}

This work was supported by the R\&D program of MOTIE/ KEIT [10054578, development of core technology for 9-axis smart motion sensor]. 


\section{References}

[1] N. Yazdi, F. Ayazi, and K. Najafi, "Micromachined inertial sensors," Proceedings of the IEEE, vol. 86, no. 8, pp. 1640-1658, 1998.

[2] M. Zaman, A. Sharma, B. Amini, and F. Ayazi, "Towards inertial grade vibratory microgyros: a high-Q in-plane silicon-on-insulator tuning fork device," in Proceedings of the Solid-State Sensor, Actuator and Microsystems Workshop, pp. 6-10, Hilton Head Island, SC, USA, 2004.

[3] R. Oboe, R. Antonello, E. Lasalandra, G. S. Durante, and L. Prandi, "Control of a Z-axis MEMS vibrational gyroscope," IEEE/ASME Transactions on Mechatronics, vol. 10, no. 4, pp. 364-370, 2005.

[4] M. F. Zaman, A. Sharma, and F. Ayazi, "High performance matched-mode tuning fork gyroscope," in Proceedings of the 19th IEEE International Conference on Micro Electro Mechanical Systems (MEMS '06), pp. 66-69, Istanbul, Turkey, 2006.

[5] T. Juneau, A. P. Pisano, and J. H. Smith, "Dual axis operation of a micromachined rate gyroscope," in Proceedings of the 9th International Conference on Solid-state Sensors and Actuators (TRANSDUCERS '97), vol. 2, pp. 883-886, IEEE, Chicago, Ill, USA, 1997.

[6] D. Xia, C. Yu, and L. Kong, "The development of micromachined gyroscope structure and circuitry technology," Sensors (Switzerland), vol. 14, no. 1, pp. 1394-1473, 2014.

[7] M. Traechtler, T. Link, J. Dehnert, J. Auber, P. Nommensen, and Y. Manoli, "Novel 3-axis gyroscope on a single chip using SOI-technology," in Proceedings of the 6th IEEE Conference on SENSORS (IEEE SENSORS '07), pp. 124-127, Atlanta, Ga, USA, October 2007.

[8] Y. Xu, S. Wang, Y. Wang, Q. Shi, and X. Ji, "A monolithic triaxial micromachined silicon capacitive gyroscope," in Proceedings of the 1st IEEE International Conference on Nano/Micro Engineered and Molecular Systems (NEMS '06), vol. 18, pp. 213-217, IEEE, Zhuhai, China, January 2006.

[9] A. M. Shkel, "Micromachined gyroscopes: challenges, design solutions, and opportunities," in Proceedings of the SPIE's 8th Annual International Symposium on Smart Structures and Materials, pp. 74-85, International Society for Optics and Photonics, Newport Beach, Calif, USA, March 2001.

[10] C. Acar, "High-performance 6-Axis MEMS inertial sensor based on through-silicon via technology," in Proceedings of the 2016 IEEE International Symposium on Inertial Sensors and Systems, vol. 22, pp. 62-65, IEEE, Laguna Beach, Calif, USA, 2016.

[11] B. Vigna, "Tri-axial MEMS gyroscopes and six degree-of-freedom motion sensors," in Proceedings of the IEEE International Electron Devices Meeting (IEDM '11), p. 29.1.3, Washington, DC, USA, December 2011.

[12] A. N. Ozan and J. Seeger, inventors; Invensense, Inc., assignee, Gyroscope self test by applying rotation on coriolis sense mass, United States patent application US 13/862,352, April 2013.

[13] G. Cazzaniga and L. Coronato, inventors; STMicroelectronics Srl, assignee. Integrated microelectromechanical gyroscope with improved driving structure, United States patent US 8,950,257, February 2015.

[14] S. T. Microelectronics, "Everything about STMicroelectronics'3-Axis Digital MEMS Gyroscopes," Technical Article TA0343, STMicroelectronics, 2011.

[15] A. Rocchi, "Maxim Integrated Products, Inc., assignee. MEMS gyroscope for detecting rotational motions about an X-, Y-, and/or Z-axis," United States patent US 8,789,416, July 2014.
[16] Y. Jeon, H. Kwon, H. C. Kim, and S. W. Kim, "Design and development of a 3-axis micro gyroscope with vibratory ring springs," Procedia Engineering, vol. 87, pp. 975-978, 2014.

[17] K. Jia, "Multiple sense axis mems gyroscope having a single drive mode," Google Patents, 2015.

[18] S. Sonmezoglu, P. Taheri-Tehrani, C. Valzasina et al., "Singlestructure micromachined three-axis gyroscope with reduced drive-force coupling," IEEE Electron Device Letters, vol. 36, no. 9, pp. 953-956, 2015.

[19] S. Wisher, P. Shao, A. Norouzpour-Shirazi et al., "A high-frequency epitaxially encapsulated single-drive quad-mass tri-axial resonant tuning fork gyroscope," in Proceedings of the IEEE 29th International Conference on Micro Electro Mechanical Systems (MEMS '16), pp. 930-933, IEEE, Shanghai, China, January 2016.

[20] A. N. Radwan, R. S. Almikhlafi, and M. S. M. Soliman, "Performance comparison of commonly used MEMS springs: double-folded, U-shaped and fish-hook," in Proceedings of the Saudi International Electronics, Communications and Photonics Conference (SIECPC '13), pp. 1-6, IEEE, April 2013.

[21] S. V. Iyer and T. Mukherjee, "Numerical spring models for behavioral simulation of MEMS inertial sensors," in Proceedings of the Design, Test, Integration, and Packaging of MEMS/MOEMS, pp. 55-62, May 2000.

[22] M. A. Shah, F. Iqbal, and B. L. Lee, "Simulation of a dual axis MEMS seismometer for building monitoring system," in Proceedings of the COMSOL Conference, Boston, Mass, USA, 2015.

[23] M. A. Shah, F. Iqbal, and B. L. Lee, "Design and analysis of a single-structure three-axis MEMS gyroscope with improved coupling spring," in Proceedings of the 11th Annual IEEE International Conference on Nano/Micro Engineered and Molecular Systems (IEEE-NEMS '16), Matsushima Bay and Sendai MEMS City, Japan, April 2016.

[24] P. P. Kwok, Fluid effects in vibrating micromachined structure [Ph.D. thesis], Massachusetts Institute of Technology, 1999.

[25] J. Cui, X. Z. Chi, H. T. Ding, L. T. Lin, Z. C. Yang, and G. Z. Yan, "Transient response and stability of the AGC-PI closedloop controlled MEMS vibratory gyroscopes," Journal of Micromechanics and Microengineering, vol. 19, no. 12, Article ID 125015, 2009. 


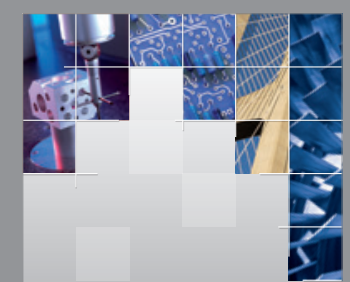

\section{Enfincering}
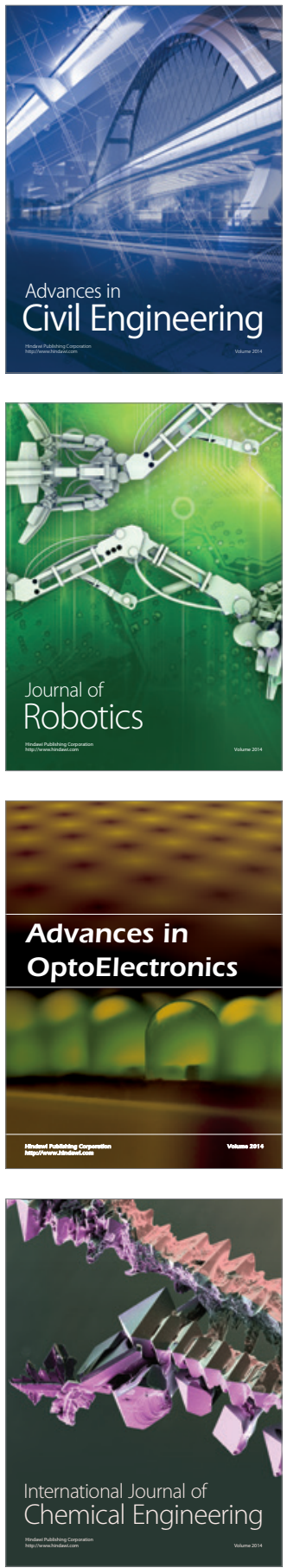

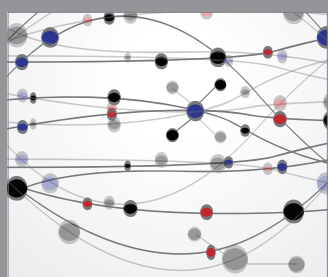

The Scientific World Journal

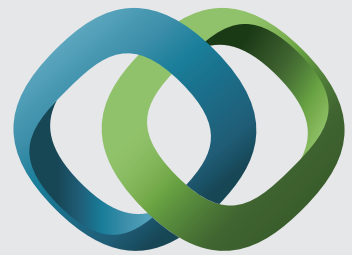

\section{Hindawi}

Submit your manuscripts at

http://www.hindawi.com
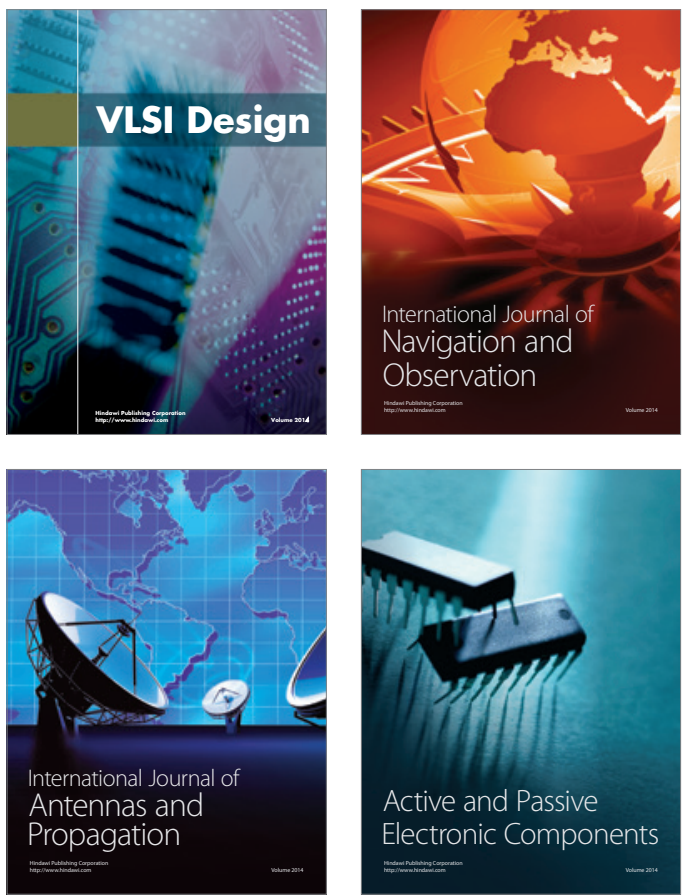
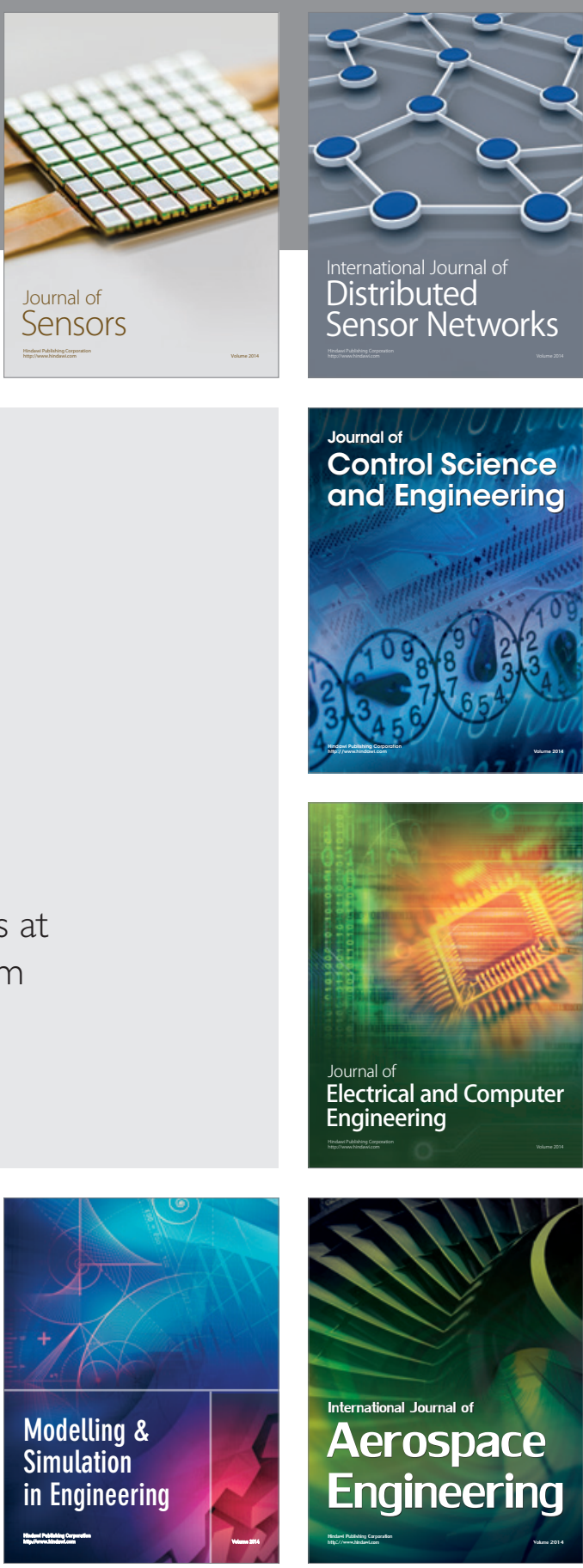

International Journal of

Distributed

Sensor Networks

Journal of

Control Science

and Engineering
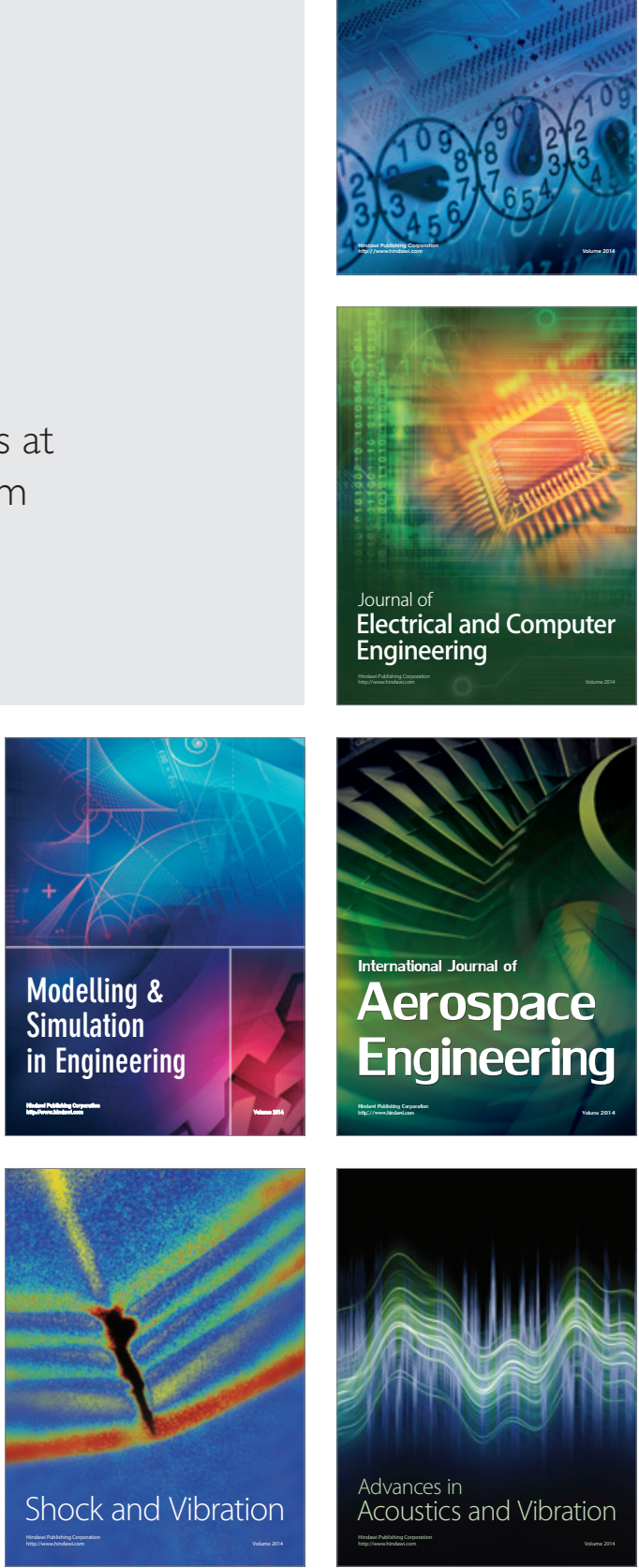